\title{
BEYOND INTERPOLATION: OPTIMAL RECONSTRUCTION BY QUASI-INTERPOLATION
}

\section{Laurent Condat ${ }^{*}$}

\author{
Laboratoire LIS - Fédération ELESA \\ Av. Félix Viallet \\ 38031 Grenoble cedex, France \\ E-mail: laurent.condat@lis.inpg.fr
}

\begin{abstract}
We investigate the use of quasi-interpolating approximation schemes, to construct an estimate of an unknown function from its given discrete samples. We show theoretically and with practical experiments that such methods perform better than classical interpolation, for the same computation cost.
\end{abstract}

\section{INTRODUCTION}

Resampling plays a central role in signal and image processing. This operation is required whenever a signal has to be evaluated at some locations where it is unknown, e.g. in order to perform sampling rate conversions or to rescale, translate or rotate digital images. In all cases, the problem amounts to recovering, from known samples, a continuouslydefined estimate of an unknown function, which is then resampled on a new grid. Shannon's theory [1] provides an exact way to recover a function from its samples, assuming it is bandlimited and sampled accordingly. However, the slow decay of the sinc interpolator, and the ringing artifacts it may introduce, prevent its practical use. Instead, practitioneers rely on convolution with more localized kernel functions having compact support, like bilinear, bicubic, or cubic spline interpolators [2].

Once the kernel has been chosen, interpolation consists in computing the unique function, in the reconstruction space, that goes through the known samples. This paper aims at showing that this is not the best way for estimating a function from its given discrete samples. Instead, we put forward the use of quasi-interpolating schemes, which are well known among approximation theoreticians (e.g. [3]), but have found small support in practical applications. In this paper, we present some elements of approximation theory which are exploited to design efficient prefilters, that have to be applied to the data prior to continuous model fitting.

\footnotetext{
* This work was performed during the first author's visit at BIG, EPFL supported by Région Rhône-Alpes (EURODOC grant).
}

Thierry Blu, Michael Unser

\author{
Biomedical Imaging Group, STI/IOA \\ Swiss Federal Institute of Technology Lausanne \\ CH-1015 Lausanne EPFL, Switzerland
}

\section{APPROXIMATION IN SHIFT-INVARIANT SPACES}

\subsection{Formulation of the problem}

Given the discrete samples $f_{k}=f(k), \quad k \in \mathbb{Z}$ of an unknown function $f(x) \in L_{2}(\mathbb{R})$, we want to find the function $f_{\text {app }}(x)$ which best approximates $f(x)$, that is which minimizes $\left\|f_{\text {app }}-f\right\|_{L_{2}}^{2}=\int_{\mathbb{R}}\left(f_{\text {app }}(x)-f(x)\right)^{2} d x$.

In the sequel, we denote $H(z)=\sum_{k \in \mathbb{Z}} h_{k} z^{-k}$ the $z$ transform of any digital filter $\left(h_{k}\right)$ and $\hat{h}(\omega)=H\left(e^{j \omega}\right)$ its Fourier transform. We also define the Fourier transform of any function $g(x) \in L_{2}$ by $\hat{g}(\omega)=\int_{\mathbb{R}} g(x) e^{-j \omega x} d x$.

If $f(x)$ is bandlimited, i.e. $\hat{f}(\omega)=0,|\omega| \geq \pi$, Shannon's theorem dictates that $f(x)$ can be recovered exactly:

$$
f(x)=\sum_{k \in \mathbb{Z}} f_{k} \operatorname{sinc}(x-k) \quad \forall x \in \mathbb{R}
$$

where $\operatorname{sinc}(x)=\frac{\sin (\pi x)}{\pi x}$. However, this method is not used in practice because of the slow decay of $\operatorname{sinc}(x)$, which makes Eqn. (1) sensitive to noise. Moreover, if $f(x)$ is not bandlimited, the reconstruction is not exact and ringing artifacts may appear. Instead, we look for a reconstruction in a linear shift-invariant space $V_{\varphi}=\operatorname{Span}\left(\{\varphi(x-k)\}_{k \in \mathbb{Z}}\right)$ generated by the translates of a template function $\varphi(x)$ [4]:

$$
f_{\text {app }}(x)=\sum_{k \in \mathbb{Z}} c_{k} \varphi(x-k) \quad \forall x \in \mathbb{R}
$$

where the coefficients $\left(c_{k}\right)$ are obtained by discrete filtering:

$$
\left(c_{k}\right)=\left(f_{k}\right) *\left(p_{k}\right) .
$$

In the following, we suppose that $\varphi$ has compact support, which makes Eqn. (2) computationally attractive. The method is equivalent to performing an expansion like in Eqn. (1): indeed, if we define the kernel $\varphi_{\text {eq }}(x)$ by 


$$
\varphi_{\mathrm{eq}}(x)=\sum_{k \in \mathbb{Z}} p_{k} \varphi(x-k) \Leftrightarrow \hat{\varphi}_{\mathrm{eq}}(\omega)=\hat{p}(\omega) \hat{\varphi}(\omega)
$$

then $f_{\text {app }}(x)=\sum f_{k} \varphi_{\text {eq }}(x-k)$. Shannon's reconstruction is retrieved if $\varphi(x)=\varphi_{\mathrm{eq}}(x)=\operatorname{sinc}(x)$ and $P(z)=1$.

\section{2. interpolation and quasi-interpolation}

One speaks about interpolation when $f_{\text {app }}(x)$ is required to verify $f_{\text {app }}(k)=f_{k}$. Then $\varphi_{\mathrm{eq}}(x)$ is an interpolator, i.e. $\varphi_{\mathrm{eq}}(k)=\{1$ if $k=0,0$ else $\}$. In this case, the prefilter $\left(p_{k}\right)$ in Eqn. (3) has to be chosen as

$$
P(z)=\frac{1}{\sum_{k \in \mathbb{Z}} \varphi(k) z^{-k}} .
$$

On the other hand, the approximation scheme is quasiinterpolating of order $N$ if the interpolation condition holds only when $f(x)$ is a polynomial of degree $\leq N-1$. This is a weaker constraint than interpolation, which adds freedom in the choice of $\left(p_{k}\right)$. We will show that this filter can be chosen so that the error $\left\|f_{\text {app }}-f\right\|_{L_{2}}$ is reduced in comparison with the interpolating case. The next section shows how to evaluate quantitatively this error.

\section{EVALUATION OF THE APPROXIMATION ERROR}

A remarkable result from [4] is that the error $\left\|f_{\text {app }}-f\right\|_{L_{2}}$ can be predicted very accurately by the quantity

$$
\varepsilon_{f}=\sqrt{\frac{1}{2 \pi} \int|\hat{f}(\omega)|^{2} E(\omega) d \omega .}
$$

The error kernel $E(\omega)$ characterizes the approximation method. Let us define $\varphi_{d}$, the dual function of $\varphi$, by

$$
\hat{\varphi}_{d}(\omega)=\frac{\hat{\varphi}(\omega)}{\hat{a}_{\varphi}(\omega)}
$$

where the discrete autocorrelation filter $a_{\varphi}=\left(a_{k}\right)$ is defined by $a_{k}=\int_{\mathbb{R}} \varphi(x) \varphi(x-k)$. Then the error kernel can be written as (the star is for complex conjugation):

$$
E(\omega)=\underbrace{1-\frac{|\hat{\varphi}(\omega)|^{2}}{\hat{a}_{\varphi}(\omega)}}_{E_{\min }(\omega)}+\underbrace{\hat{a}_{\varphi}(\omega)\left|\hat{p}(\omega)^{*}-\hat{\varphi}_{d}(\omega)\right|^{2}}_{E_{\mathrm{res}}(\omega)} .
$$

$E_{\min }(\omega)$ is a lower bound for $E(\omega)$, describing the error when $f_{\text {app }}$ is the orthogonal projection of $f$ upon $V_{\varphi}$, which is optimal but unattainable in our case (because $f$ is unknown). We first have to choose $\varphi(x)$ so that $E_{\min }(\omega)$ is minimized. As in practice most signals, and particularly images, are essentially lowpass, $E_{\min }(\omega)$ must vanish as much as possible near $\omega=0$. More specifically, we enforce

$$
E_{\min }(\omega)=O\left(\omega^{2 L}\right)
$$

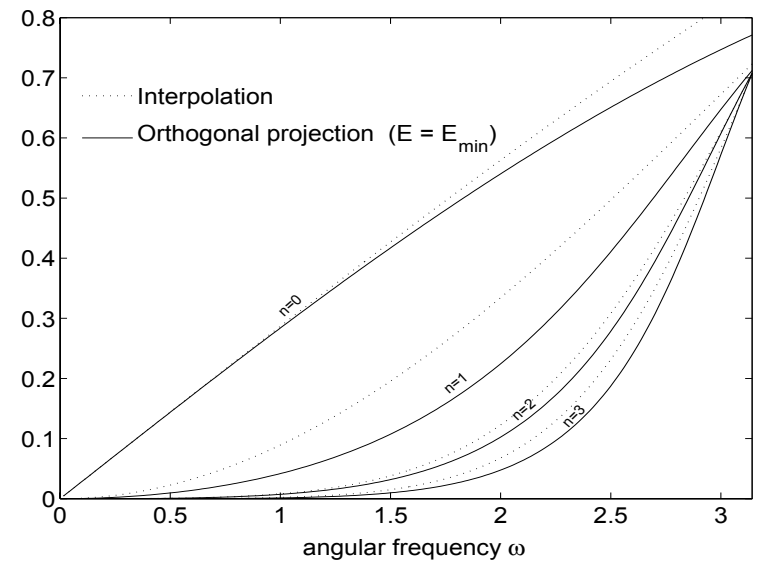

Fig. 1. $\sqrt{E(\omega)}$ when $\varphi=\beta^{n}$ is a centered B-spline.

where the integer $L$ should be chosen as large as possible. This is equivalent to the well-known Strang-Fix conditions [5]:

$$
\hat{\varphi}(0) \neq 0 \text { and } \hat{\varphi}^{(k)}(2 n \pi)=0 \text { for }\left\{\begin{array}{l}
n \neq 0 \\
k=0 \ldots L-1
\end{array}\right.
$$

in which case $\varphi$ is said to have approximation order $L$. Because the support of $\varphi$ has a size $T \geq L$, with equality iff $\varphi$ is a MOMS [6], $L$ has to be chosen small enough so as to keep a reasonable computation cost.

In Fig. 1, we depict $\sqrt{E_{\min }(\omega)}$ and $\sqrt{E(\omega)}$ in the interpolating case described by Eqn. (5). $\varphi(x)$ is chosen as the centered B-spline $\beta^{n}(x)$ of degree $n$, which is a MOMS with order $L=n+1$. It satisfies $\hat{\beta}^{n}(\omega)=\left(\frac{\sin (\omega / 2)}{\omega / 2}\right)^{n+1}$ and $a_{k}=\beta^{2 n+1}(k)$. As we can see, the approximation quality depends directly on $L$, as confirmed by experiments in [2]. The difference between approximation by orthogonal projection and by interpolation is significant, particularly for a small order $L$. We now detail how this difference can be reduced if we use a non-interpolating approximation.

\section{DESIGN OF OPTIMAL PREFILTERS}

\subsection{Quasi-interpolating all-pole filters}

If the interpolation constraint is relaxed, the filter $\left(p_{k}\right)$ can be chosen so that $E_{\text {res }}(\omega)$ is arbitrarily small in the Nyquist band $\omega \in]-\pi, \pi[$, especially around $\omega=0$. We want that

$$
E(\omega) \sim E_{\min }(\omega)
$$

in order for $f_{\text {app }}(x)$ to be as well behaved as the optimal orthogonal projection of $f$ upon $V_{\varphi}$, near $\omega=0$. This amounts to require that

$$
\hat{p}(\omega)=\hat{\varphi}_{d}(\omega)^{*}+O\left(\omega^{N}\right)
$$


with $N \geq L+1$. As soon as $N \geq L$, it is known that the approximation scheme is quasi-interpolating of order $L$ [4].

In [4], the shortest FIR filters satisfying $N=L+1$ in Eqn. (12) were designed. In this paper, we propose instead to use all-pole IIR filters, which arise naturally in this context because the interpolation prefilters are of this type (see Eqn. (5)).

It is essential to notice that a convolution with an IIR all-pole filter $1 / H(z)$ can be performed by a fast algorithm, which takes essentially the same time as a FIR convolution by $H(z)$ [7]. When using the resampling algorithm defined by Eqns (2),(3), most of the time is consumed by the multiple evaluations of $\varphi(x)$, while the prefiltering step has a minor contribution, as confirmed in Tab. 2. That is why $\varphi$ must have minimal support.

So, we are looking for a filter with the form $P(z)=$ $1 / Q(z)$, where $\left(q_{k}\right)$ is a FIR filter, so that Eqn. (12) is satisfied. If $\varphi(x)$ is symmetric, we choose a symmetric filter, so that the whole process has linear phase. Note that Eqn. (10) implies that $\hat{a}_{\varphi}(\omega)=|\hat{\varphi}(\omega)|^{2}+O\left(\omega^{2 L}\right)$. Then, if $N \leq 2 L$, Eqn. (12) can be rewritten as

$$
\hat{q}(\omega)=\hat{\varphi}(\omega)+O\left(\omega^{N}\right) .
$$

\subsection{Practical examples}

As an example, we give in Tab. 1 the prefilters obtained for spline approximation using $\varphi(x)=\beta^{n}(x)$ with the most used degrees $n=0 \ldots 3$. We choose $N=L+1$ in Eqn. (12), or $N=L+2$ in the case where the interpolating prefilter in Eqn. (5) already satisfies Eqn. (12) with $N=$ $L+1$, which is the case for even values of $n$.

Let us detail the design of a prefilter for the piecewise linear approximation corresponding to $\varphi=\beta^{1}$, which has approximation order $L=2$. We have the development:

$$
\hat{\varphi}(\omega)=1-\frac{1}{12} \omega^{2}+O\left(\omega^{4}\right) .
$$

We are looking for a filter $\hat{q}(\omega)=1-u+u \cos (\omega)$ such that $\hat{q}(\omega)=\hat{\varphi}(\omega)+O\left(\omega^{3}\right)$. This yields $u=\frac{1}{6}$, and $Q(z)=\frac{1}{12} z^{-1}+\frac{5}{6}+\frac{1}{12} z$.

On Fig. 2 we show the spectrum of the corresponding quasi-interpolator (see Eqn. (4)), which is closer to the ideal

\begin{tabular}{|c|c|}
\hline$\varphi(x)$ & $Q(z)=1 / P(z)$ \\
\hline$\beta^{0}(x)$ & $-\frac{1}{24} z^{-1}+\frac{13}{12}-\frac{1}{24} z$ \\
$\beta^{1}(x)$ & $\frac{1}{12} z^{-1}+\frac{5}{6}+\frac{1}{12} z$ \\
$\beta^{2}(x)$ & $-\frac{7}{1920} z^{-2}+\frac{67}{480} z^{-1}+\frac{233}{320}+\frac{67}{480} z-\frac{7}{1920} z^{2}$ \\
$\beta^{3}(x)$ & $-\frac{1}{720} z^{-2}+\frac{31}{180} z^{-1}+\frac{79}{120}+\frac{31}{180} z-\frac{1}{720} z^{2}$ \\
\hline
\end{tabular}

Table 1. Proposed prefilters for spline quasi-interpolation.

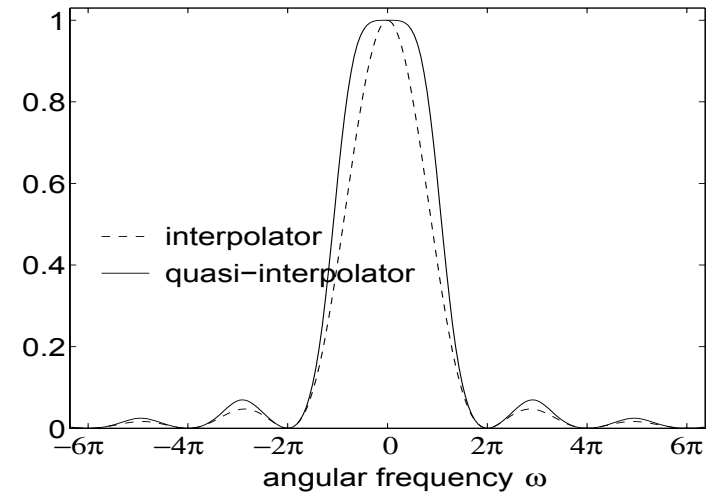

Fig. 2. $\hat{\varphi}_{\text {eq }}(\omega)$ corresponding to piecewise-linear interpolation and quasi-interpolation.

lowpass filter than the interpolator $\hat{\beta}^{1}(\omega)$. Then its use will introduce much less blurring, as confirmed in Fig. 3.

\section{EXPERIMENTS}

To demonstrate the effectiveness of the proposed approach, we perform 17 rotations by a $2 \pi / 17$ angle on standard images, using different interpolators and quasi-interpolators. The process is applied in a separable way, and we use classical mirror boundary conditions.

The results are shown in Tab. 2. In the first table, the first column is for bilinear interpolation $\left(\varphi=\beta^{1}, P(z)=\right.$ $1)$, the second one is for shifted interpolation, an alternative method proposed in [8] with $\varphi(x)=\beta^{1}(x-\tau), \tau=$ $\frac{1}{2}\left(1-\frac{\sqrt{3}}{3}\right)$ and $P(z)=1 /\left(1-\tau+\tau z^{-1}\right)$. The third and

\begin{tabular}{|c|c|c|c|c|}
\hline (L=2) & int. & int. shift. & quasi. FIR & quasi. IIR \\
\hline Lena & 29.40 & 34.87 & 35.47 & 36.81 \\
Barbara & 23.84 & 25.96 & 26.13 & 27.32 \\
Baboon & 21.98 & 24.40 & 25.17 & 26.23 \\
Boat & 26.33 & 30.59 & 31.37 & 32.52 \\
Camera & 23.29 & 26.54 & 27.49 & 28.64 \\
Peppers & 28.89 & 33.08 & 34.36 & 35.41 \\
\hline time & $1 \mathrm{U}$ & $1.05 \mathrm{U}$ & $1.1 \mathrm{U}$ & $1.1 \mathrm{U}$ \\
\hline & Bicubic int. & Sp-3 int. & Sp-3 quasi. \\
\hline Lena & 35.29 & 38.69 & 39.81 \\
Barbara & 25.95 & 28.99 & 30.55 \\
Baboon & 25.01 & 27.63 & 28.64 \\
Boat & 31.21 & 34.07 & 35.00 \\
Camera & 27.33 & 30.18 & 31.23 \\
Peppers & 34.22 & 36.83 & 37.64 \\
\hline time & $2.3 \mathrm{U}$ & $2.6 \mathrm{U}$ & $2.7 \mathrm{U}$ \\
\hline
\end{tabular}

Table 2. PSNR after 17 rotations by $2 \pi / 17$ on images, using interpolating and quasi-interpolating methods. 
fourth columns are for bilinear quasi-interpolation, with the prefilters being respectively $P(z)=\frac{-1}{12} z^{-1}+\frac{7}{6}+\frac{-1}{12} z$ proposed in [4] and our new IIR filter proposed in Tab. 1.

The second table shows the results for cubic spline (i.e. $\varphi=\beta^{3}$, denoted Sp-3) interpolation and proposed quasiinterpolation. The well-known bicubic interpolation proposed by Keys [9] (having order $L=3$ ) is also evaluated.

As we see from these numerical results as well as in Fig. 3, the proposed quasi-interpolators provide significant improvements over their interpolating counterparts, with almost no increase in computation time, given in arbitrary unit. Bilinear quasi-interpolation performs better than bicubic interpolation, and is twice faster. These experiments validate the theoretical design and show the relevancy to minimize the error kernel $E(\omega)$.

\section{CONCLUSION}

In this work, we demonstrated the relevance of using quasiinterpolation for resampling purpose. We designed IIR optimal filters allowing approximation with essentially the same performance as the optimal least-squares solution. The gain in comparison with classical interpolation has been shown theoretically using the error kernel, and practically by rotation experiments, which validate the proposed approach.

\section{REFERENCES}

[1] C. E. Shannon, "Communication in the presence of noise," Proc. of the Inst. of Radio Eng., vol. 37, no. 1, pp. 10-21, 1949.

[2] P. Thévenaz, T. Blu, and M. Unser, "Interpolation revisited," IEEE Trans. Med. Imag., vol. 19, no. 7, pp. 739-758, 2000.

[3] C. de Boor and G. Fix, "Spline approximation by quasiinterpolants," J. Approx. Theory, vol. 8, pp. 19-45, 1973.

[4] T. Blu and M. Unser, "Quantitative Fourier analysis of approximation techniques: Part I-interpolators and projectors and part II-wavelets," IEEE Trans. Sig. Proc., vol. 47, no. 10, pp. 2783-2806, Oct. 1999.

[5] G. Strang and G. Fix, "A Fourier analysis of the finite element variational method," in Constructive aspect of functional analysis, Rome, Italy: Cremonese, 1971, pp. 796-830.

[6] T. Blu, P. Thévenaz, and M. Unser, "Moms: Maximal-order interpolation of minimal support," IEEE Trans. Image Proc., vol. 10, no. 7, pp. 1069-1080, Jul. 2001.

[7] M. Unser, "Splines: A perfect fit for signal and image processing," IEEE Signal Proc. Mag., vol. 16, no. 6, Nov. 1999.

[8] T. Blu, P. Thévenaz, and M. Unser, "Linear interpolation revitalized," IEEE Trans. Image Proc., vol. 13, no. 5, pp. 710-719, May 2004.

[9] R. G. Keys, "Cubic convolution interpolation for digital image processing," IEEE Trans. Acoust., Speech, Sig. Proc., vol. 29, pp. 1153-1160, 1981.

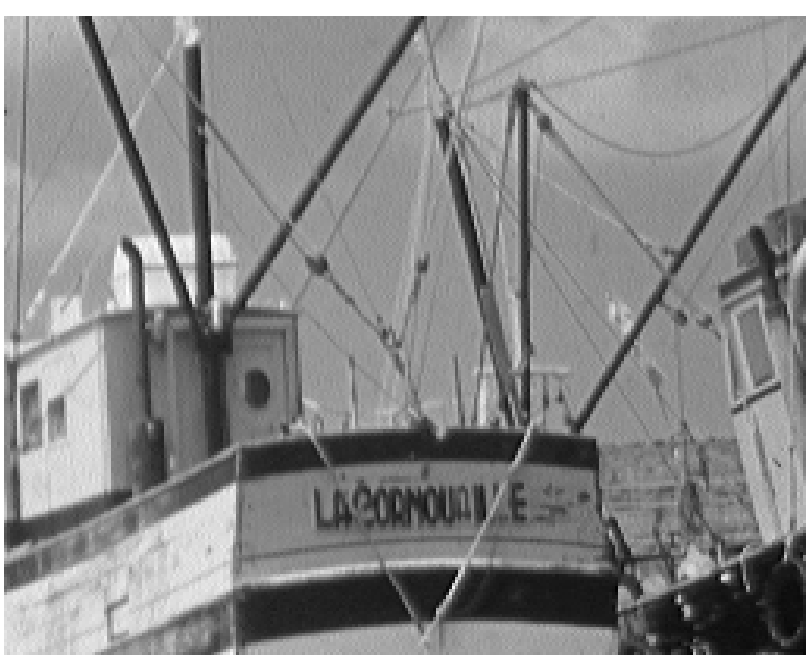

Part of the original boat image

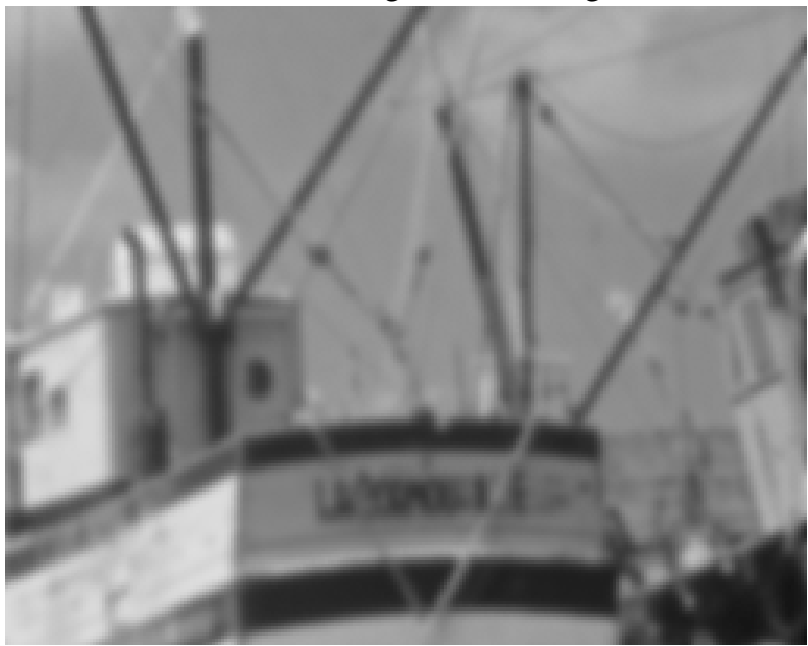

bilinear interpolation

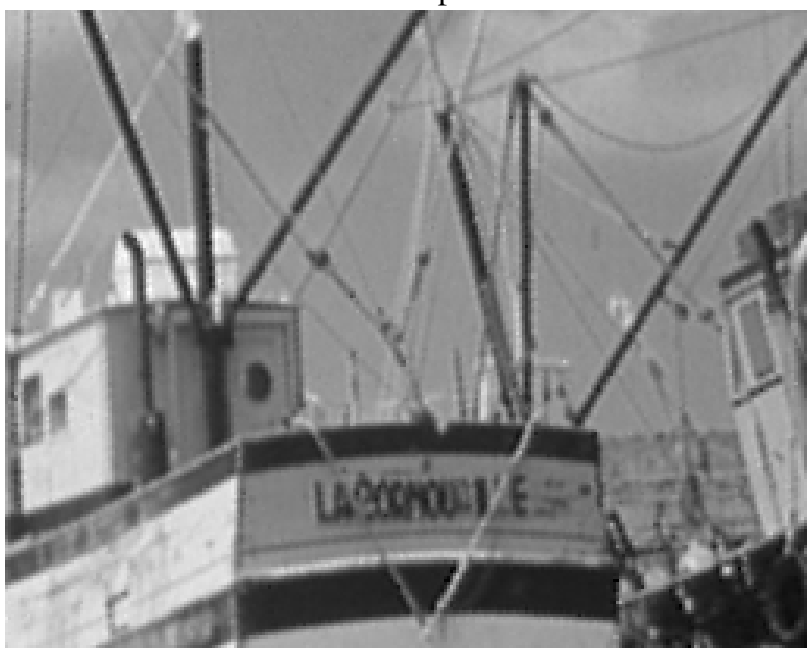

bilinear quasi-interpolation

Fig. 3. Part of the image obtained after 17 rotations by $2 \pi / 17$ with bilinear interpolation and quasi-interpolation. 